\title{
LIMITING CASE HARDY INEQUALITIES ON THE SPHERE
}

\section{Ahmed A. AbDelhakim}

Abstract. We give sharp limiting case Hardy inequalities on the sphere $\mathbb{S}^{2}$ and show that their optimal constants are unattainable by any $f \in H^{1}\left(\mathbb{S}^{2}\right) \backslash\{0\}$. The singularity of the problem is related to the geodesic distance from a point on the sphere.

Mathematics subject classification (2010): 26D10, 35A23, 46E35. folds.

Keywords and phrases: Critical Hardy inequality, sharp constant, 2-sphere, Sobolev spaces on mani-

\section{REFERENCES}

[1] A. Balinsky, W. D. Evans, R. T. Lewis, The analysis and geometry of Hardy's inequality, Springer, New York 2015.

[2] J. BYeOn, F. TAKAhAShI, Hardy's inequality in a limiting case on general bounded domains, arXiv: 1707.04018, 2017.

[3] JÜrgen EIChHorn, Global analysis on open manifolds, Nova Science Publishers, Inc., New York, 2007.

[4] N. Ioku, M. Ishiwata, A scale invariant form of a critical Hardy inequality, International Mathematics Research Notices 18 (2015), 8830-8846.

[5] N. IOKU, M. IsHIWATA AND T. OZAWA, Sharp remainder of a critical Hardy inequality, Archiv der Mathematik 106 (2016), 65-71.

[6] S. Machinara, T. OZAWA AND H. WAdade, Hardy type inequalities on balls, Tohoku Mathematical Journal 65 (2013), No. 3, 321-330.

[7] M. RuZhansky AND D. Suragan, Critical Hardy inequalities, arXiv: 1602.04809, 2016.

[8] M. SANo, F. TAKAHASHI, Scale invariance structures of the critical and the subcritical Hardy inequalities and their improvements, Calculus of variations and partial differential equations, (2017) 56: 69.

[9] X. Sun, F. PAN, Hardy type inequalities on the sphere, J. Inequalities and Applications, (2017) 2017: 148.

[10] F. TAKAHASHI, A simple proof of Hardy's inequality in a limiting case, Archiv der Mathematik 104 (2015), 1, 77-82.

[11] Y. XIAO, Some Hardy inequalities on the sphere, J. Math. Inequal. 10 (2016), 793-805. 